\title{
Autologous Hematopoietic Stem Cell Transplant Recipient
}

National Cancer Institute

\section{Source}

National Cancer Institute. Autologous Hematopoietic Stem Cell Transplant Recipient. NCI

Thesaurus. Code C153350.

A person receiving an autologous hematopoietic stem cell transplant. 